\title{
The association between dietary antioxidants intakes and the risk of cardiovascular disease: Tehran Lipid and Glucose Study
}

\section{Parvin Mirmiran}

Shaheed Beheshti University of Medical Sciences

\section{Zohreh Esfandiar}

Shaheed Beheshti University of Medical Sciences

Firoozeh Hosseini-Esfahani ( $\nabla$ f.hosseini@sbmu.ac.ir)

Shaheed Beheshti University of Medical Sciences Research Institute for Gastroenterology and Liver Diseases https://orcid.org/0000-0002-9697-0081

somayeh Hosseinpour-Niazi

Shaheed Beheshti University of Medical Sciences

Fereidoun Azizi

Shaheed Beheshti University of Medical Sciences

\section{Research}

Keywords: vitamin A, vitamin E, vitamin C, zinc, antioxidant, cardiovascular disease

Posted Date: February 18th, 2020

DOI: https://doi.org/10.21203/rs.2.18583/v2

License: (c) (i) This work is licensed under a Creative Commons Attribution 4.0 International License. Read Full License 


\section{Abstract}

Aim: This study aimed at investigating the association between daily consumption of dietary vitamins $A, E$, and $C$, and zinc and the incidence of cardiovascular disease (CVD). Methods: Eligible adults ( $n=5102)$ were selected from the participants of the Tehran Lipid and Glucose Study with an average follow-up of 5.3 years. Dietary intakes were assessed using a valid and reliable semi-quantitative food frequency questionnaire. Anthropometrics and biochemical variables were evaluated at baseline and follow-up examinations. Multivariate Cox proportional hazard regression models were used to estimate the development of CVD associated with total intakes of vitamins A, E, and C, and zinc. Results: This study was conducted on 2,253 men and 2,849 women aged $47.0 \pm 11.6$ and $45.6 \pm 10.5$ years, respectively. The main sources of dietary vitamins $A, E$, and $C$ and zinc were fruits, vegetables, and legumes. Risk of CVD decreased from quartile 1 to quartile 4 for vitamin $E$ intake $(\mathrm{HR}(95 \% \mathrm{Cl}): 1.00,0.91,0.77$, and 0.57 ; $\mathrm{P}$ trend $=0.03)$. The association between risk of CVD and the quartiles of vitamins $\mathrm{A}$ and $\mathrm{C}$ and zinc intake was not significant. Conclusion : Our study suggested an inverse association between vitamin E intake and the risk of CVD. The results emphasized a potential protective role of its dietary sources in the prevention of CVD.

\section{Introduction}

Cardiovascular disease (CVD), the leading cause of death worldwide, is the collective term/compound name for disorders afflicting the blood vessels and heart that accounts for 17.9 million deaths in 2016 (1). Inflammation and enhanced oxidative stress have been shown as fundamental risk factors in the onset and progression of CVD (2). Chronic inflammatory conditions attenuate blood levels of antioxidants because of the continuous generation of elevated levels of reactive oxygen species (ROS). A sufficient intake of antioxidants is also suggested to beneficially interfere with CVD by quenching ROS (3).

Antioxidant vitamins and minerals, such as vitamins $A, E$, and $C$, and zinc may slow the development and progression of CVD $(4,5)$. Observational epidemiological studies have suggested that higher circulating concentrations of vitamin E were associated with a lower risk of CVD (6); therefore, these findings indicate the need for additional and more accurate clinical investigations in this area.

Vitamin C is a major water-soluble antioxidant in plasma, and observational studies have shown inverse associations between dietary vitamin C and CVD outcomes $(7,8)$; however, in several large randomized controlled trials, the effects of vitamin $C$ to prevent CVD has not been confirmed. Although the antioxidant potential of vitamin A was first determined in 1932 (9), limited data is available regarding the association between vitamin A and CVD (10). Zinc is an essential trace metal with antioxidant and anti-inflammatory activities; insufficient intake of zinc has persistently been reported in CVD patients (11). Trace element analysis of hair showed that patients with CVD had lower levels of zinc (12). A 6-year follow-up cohort study on CVDs and dietary zinc intake reported no association between CVD and total zinc intake (13); therefore, further investigations, as well as additional evidence obtained by observational studies are required.

Accordingly, studies on the associations between CVD and the antioxidant vitamins and minerals, such as vitamins $A, E$, and $C$ and zinc can form the local dietary guidelines regarding the nutritional value of food groups; therefore, due to the limited evidence in Asian population, it is needed to investigate the risk of CVD by 
dietary antioxidant vitamins and minerals intakes (14). This study aimed at prospectively evaluating the association between dietary antioxidant intakes (vitamins A, E, and C and zinc) and CVD in adults in Tehran, Iran.

\section{Methods}

\section{Study population:}

In this cohort study, the subjects were selected from participants of the Tehran Lipid and Glucose Study (TLGS), a population-based prospective study performed to determine the risk factors for non-communicable diseases among the residents of the District 13, Tehran, capital city of Iran (20). The first examination survey was performed from 1999 to 2001 on 15,005 individuals aged $\geq 3$ years, using the multistage stratified cluster random sampling, and follow-up examinations were conducted every 3 years; 2002-2005 (survey 2), 20052008 (survey 3), 2008-2011(survey 4), and 2012-2015 (survey 5) to identify recently developed diseases.

Of 14,712 individuals participating at baseline (surveys 3 and 4) , 9057 subjects were randomly selected for dietary assessment based on age- and sex-stratified random sampling, of whom 5,531 subjects aged $\geq 30$ years at baseline with completed data were included and followed until 2014. Subjects $(n=227)$ with under- or over-reporting of energy intake (<800 or $\geq 4200 \mathrm{kcal} /$ day) (15) and also those with a history of CVD ( $n=414)$ at baseline were excluded. Finally, after excluding participants who lost follow-up ( $n=15), 5,102$ subjects remained and entered the analysis (Figure 1).

All participants signed a written informed consent form prior to the research. The study was conducted based on the Declaration of Helsinki and the study protocol was accepted by the ethics committee of the Research Institute for Endocrine Sciences, Shahid Beheshti University of Medical Sciences, Tehran, Iran. All methods were performed in line with their relevant guidelines and regulations. Materials and methods were checked using the strengthening the reporting of observational studies in epidemiology (STROBE) statement.

\subsection{Dietary intake measurements}

Dietary assessment was performed by a valid and reliable 168-item semi-quantitative food frequency questionnaire (FFQ). Trained interviewers collected information on usual dietary intake through face-to-face private interviews. The frequency of eating each food item on a daily, weekly, or monthly basis was converted to daily intakes, and portion sizes were then converted into grams using household measures (16). Dietary intakes of vitamins $E, C$, and $A$, and zinc were calculated and considered in grams per week. Vitamin A consumption was considered as taking retinol and its esterified form.

\section{Physical activity}

Physical activity level was assessed using the Persian translation of the modifiable activity questionnaire (MAQ) with high reliability and moderate validity (17). The time and frequency of the light, moderate, high, and very hard intensity activity were obtained according to the list of common activities of daily living over the past year and the results were transformed into metabolic equivalent-hours/week (Met/h/week) (18).

\section{Blood pressure and anthropometric measurements}


Systolic and diastolic blood pressures (SBP and DBP) were measured twice (with a $30 \mathrm{~s}$ interval) in a sitting position after $15 \mathrm{~min}$ of rest.

The body weight was measured to the nearest $100 \mathrm{~g}$, using a digital scale (Seca 707) while the subjects were minimally clothed and without shoes. Height was measured to the nearest $0.5 \mathrm{~cm}$ by a tape measure in standing position without shoes and with shoulders in normal alignment. Waist circumference (WC) was measured with a non-flexible tape meter without any pressure to body surface at the level of the umbilicus (19) and was taken at the end of a normal expiration over light clothing. Measurements were recorded to the nearest $0.1 \mathrm{~cm}$.

\section{Laboratory assays}

Blood samples were drawn into vacutainer tubes between 7:00 and 9:00 a.m., after a 12-14-hour overnight fast in sitting position and centrifuged within 30 to $45 \mathrm{~min}$ of the collection. All biochemical analyses were performed using a Selectra 2 auto-analyzer at the TLGS research laboratory on the blood collection day. Fasting blood glucose (FBG) concentration was measured on the blood collection day using the colorimetric glucose oxidase procedure. The standard 2-h post-challenge blood glucose test was performed by oral administration of $82.5 \mathrm{~g}$ of glucose monohydrate solution (or $75 \mathrm{~g}$ of anhydrous glucose) for those who did not take glucose-lowering drugs.

High-density lipoprotein cholesterol (HDL-C) concentration was assessed after precipitation of the apolipoprotein B-containing lipoproteins by phosphotungstic acid. Total cholesterol (TC) and triglyceride (TG) were measured using the enzymatic colorimetric method. For TC assay, cholesteryl ester hydrolase was used to convert cholesteryl ester to cholesterol, which was then oxidized by cholesterol oxidase to cholesterol-4-en3-one and H2O2. TG was broken down to glycerol and free fatty acids using lipoprotein lipase and glycerol was then phosphorylated to glycerol phosphate by glycerokinase. Glycerol phosphate was then converted to dihydroxyacetone phosphate and $\mathrm{H} 2 \mathrm{O} 2$ by glycerol phosphate oxidase. The Friedewald equation (LDL-C = TC - HDL-C - TG/5) was used to calculate LDL-C concentration in samples containing TG (20).

\section{Definitions}

Details of the collection of CVD outcome data have been described elsewhere (21). Coronary heart disease (CHD) events included definite myocardial infarction (diagnostic electrocardiographic [ECG] results and biomarkers), probable myocardial infarction (positive ECG findings plus cardiac symptoms or signs plus missing biomarkers or positive ECG findings plus equivocal biomarkers), proven CHD by angiography, unstable angina pectoris (new cardiac symptoms or changing symptom patterns and positive ECG findings with normal biomarkers), and CHD mortality. CVD was defined as stroke (a new neurological deficit that lasted more than $24 \mathrm{~h}$ ), CHD events, or CVD death (a fatal stroke or fatal CHD).

Sex-specific multivariable risk functions ("general CVD" algorithms) were derived that incorporated age, TC, HDL-C, SBP, treatment for hypertension, smoking, and type 2 diabetes status (22). Hypertension was defined as $\mathrm{SBP} \geq 140, \mathrm{DBP} \geq 90 \mathrm{mmHg}$, or receiving antihypertensive drug (23).

\section{Statistical analyses}


Statistical analyses were carried out using the Statistical Package for Social Sciences (version 21.0; SPSS). A two-tailed $P$ value $<0.05$ was used to determine statistical significance. We used a Chi-square test and Student's t-test for qualitative and quantitative variables to compare the results obtained from the male and female subjects. For non-normal nutritional and biochemical variables (TG concentration), log-transformed values were used for statistical analysis. The hazard ratio (HR) and 95\% confidence interval of incident CVD were assessed using multivariable Cox proportional hazard regression models. Person-years of the follow-up were calculated for each individual between the date of study inclusion and the date of diagnosis of CVD, death, or end of the follow-up, whichever came first. Survival time for censored individuals was calculated as the interval between the first and last observation dates. The event date was considered as the middle-time between the date of follow-up visit, at which the events were diagnosed for the first time, and the most recent follow-up visit preceding the diagnosis. The incidence of CVD during the follow-up period was considered as dichotomous variables (yes/no) in the models. Vitamins $E, C$, and A, and zinc intakes were categorized into quartiles, given the first quartile as the reference. The median of each quartile was used as a continuous variable to assess the overall trends of HRs across quartiles of dietary intakes of vitamins $A, E$, and $C$ and zinc in the Cox proportional hazard regression models. Trend test was applied for evaluating dose-response effects in association studies. The proportional hazards assumption of the multivariate Cox models were assessed using Schoenfeld's global test of residuals.

The confounders were selected based on previous studies and included in the uni-variable Cox regression model. A two-tailed $\mathrm{P}$ value $<0.20$ was used for inclusion in the model (24). The Cox regression models were adjusted for age, sex, CVD risk score (continuous), family history of CVD, physical activity (continuous), dietary intakes of total energy, fiber (g/1000 Kcal) and total fat (percentage of energy).

\section{Results}

The mean age of subjects at baseline was $47.0 \pm 11.6$ and $45.6 \pm 10.5$ years in men and women, respectively. Table 1 represents the baseline characteristics of the men and women. Men were older, had worse smoking habits, greater levels of physical activity, lower body mass index and higher WC than women. In addition, men had higher values of SBP, DBP, total cholesterol, TG/HDL-ratio, and FPG except for $2 \mathrm{~h}$ - plasma glucose. Men showed greater amounts of energy and carbohydrate consumption than women. Intakes of total fat, saturated fatty acid, monounsaturated fatty acid, polyunsaturated fatty acid, fiber, vitamins A and C were higher in women compared with men.

The association between dietary intakes of vitamins E, C, and A and zinc across quartiles of food groups are presented in Table 2. Higher consumption of fruit, vegetable, legumes, refined grains, vegetable oils, fish, and poultry was associated with higher concentrations of vitamin E. Vitamin C intake was positively associated with all food groups, except for refined grains and red meat that showed a negative association with vitamin $\mathrm{C}$ intake. There was a positive association between vitamin A and quartiles of all food groups, except for red meat. Compared with those in the lower quartiles, zinc intake was significantly higher among individuals in the upper quartiles of fruit, vegetable, legumes, whole grains, refined grains, dairy, fish, and poultry intake.

HRs (95\% Cl) of CVD for quartiles of dietary antioxidants (vitamins A, E, and C, and zinc) intakes are presented in Table 3. After adjustment for potential confounders, risk of CVD decreased from quartile 1 to 
quartile 4 for vitamin E intake (HR $(95 \% \mathrm{Cl}): 1.00,0.91,0.77$, and $\left.0.57 ; P_{\text {trend }}=0.03\right)$. The association between the risk of CVD and quartiles of vitamins $A$ and $C$, and zinc intake was not significant.

Kaplan-Meier cumulative survival curves for CVD according to quartiles of vitamins A, C, and E, and zinc intakes during follow-up periods are shown in Figures 2-5. Significant differences were found in the risk of CVD between quartiles of vitamin $\mathrm{E}$ intake.

\section{Discussion}

The current investigation was a prospective cohort study, evaluating the association between dietary antioxidants (vitamins $A, E$, and $C$, and zinc) intakes and risk of CVD. Our results suggested that a higher intake of vitamin $E$ was inversely associated with the incidence of CVD.

Previous studies have found an association between dietary vitamin E intake and decreased risk of CVD in observational epidemiologic studies $(23,25-27)$. Several biological functions of vitamin $E$ are due to its antioxidant properties because of its ability to inhibit the oxidation of LDL-C and scavenge lipid radicals (28). In contrast, several large randomized controlled trials have failed to corroborate the benefits of vitamin $E$ in CVD prevention (29), which can be due to several factors, such as the time of intervention, gene polymorphisms or inherent confounding, and pathophysiological conditions in study populations. In addition, an intervention study suggested that in addition to prescribed medicine, supplemental doses of vitamin $\mathrm{E}$ should be given to ameliorate therapeutic strategies (30). However, vitamin E supplements have not been recommended by the American Heart Association to prevent CVD due to the lack of approved results; however, it recommends eating foods rich in antioxidant vitamins, especially fruits and vegetables (31).

In our study, vitamin E was associated with several food groups, which may be due to the connection between food groups, such as consuming oils (olive oil and mayonnaise) and vegetables being mixed in salads or using oils for cooking. Also, no association was found between nuts intake and vitamin E, which may be due to the nuts consumption in small quantities in our population.

Anti-CVD properties of vitamin C have not yet been fully confirmed. No significant association was found between total vitamin $\mathrm{C}$ intake (estimated by summing the vitamin $\mathrm{C}$ contribution of food items and supplements) and CVD in a cohort of Spanish university graduates. The mean of vitamin C intake (mg/day) across tertiles of vitamin C intake was 184,266 , and 387, respectively. Therefore, it concluded that the absence of significant results might be explained by the low variability in the exposure. However, vitamin $\mathrm{C}$ is a single nutrient and may not represent the synergistic effect of the whole dietary pattern (32). In contrast to our results, observational studies on the relationship between vitamin $\mathrm{C}$ and CVD risk have demonstrated an inverse association between vitamin C and CVD outcomes, especially heart failure (7) and hypertension (8). The contradiction in results can be due to differences in the definition of CVD. Vitamin $\mathrm{C}$ increases the nitric oxide bioactivity of the endothelium, which causes a decrease in blood pressure (33). Moreover, vitamin C reduces monocyte adhesion and inhibits LDL oxidation (34), which plays an important role in decreasing the risk of atherosclerosis. In addition, vitamin C keeps atheromatous plaques stable by preventing vascular smooth muscle cell apoptosis (35). 
Consistent with our results, in a meta-analysis (36), neither dietary nor supplemental vitamin A was associated with CVD risk. A large prospective study (10) indicated that among 4,117 patients with stable angina pectoris in the upper tertile of serum vitamin $A$ concentrations, serum apolipoprotein $B$ (a predictor of CVD) was associated with CVD risk. However, dietary intake of vitamin A did not correlate with serum concentrations, which seems that another mechanism, other than vitamin A intake, regulates the serum vitamin A concentrations (37). Accordingly, in the current study, no association was observed between dietary vitamin $A$ and CVD risk.

In the present study, no significant difference was found between dietary zinc intake and CVD incidence, which is consistent with a systematic review of prospective cohort studies on the association between dietary zinc intake or serum zinc levels and the incidence of CVD (38). In contrast with our result, higher total dietary zinc intake (estimated by summing the zinc contribution of food items) was associated with a greater incidence of CVD in a large longitudinal study on Australian women. Therefore, more investigations are needed to investigate the association between zinc intake from meat and other major sources because dietary guidelines recommend to reduce red meat intake and encourage consuming other sources of zinc (39). Also, more studies are needed to investigate the mechanisms of action of zinc on the pathogenesis of CVD to provide dietary zinc recommendation for preventing CVD.

The prospective design of the present study was one of its major strengths that facilitated the estimation of disease incidence without concern about reverse causality between nutrients and outcomes. In this research, the subjects' reports regarding food consumption were used, whereas multiple assessments of urine or circulating biomarkers of vitamins over time could be a more reliable approach and it was one of the limitations of this study.

Considering the observational design of the current research, some confounders (e.g. supplement intake and socioeconomic levels) were not considered. Dietary assessment was conducted at baseline only, and changes in dietary intakes were not recorded during follow-up. Moreover, some cases lost the follow-up; for example, those with a high risk of CVD were selectively excluded due to their poor mental or physical health.

\section{Conclusion}

This qualitative assessment suggested an inverse association between vitamin E intake and the risk of CVD, which emphasized the potentially protective role of its dietary sources in the prevention of CVD events.

\section{Declarations}

\section{Acknowledgments}

The authors are thankful to Niloofar Shiva and Kowsar publication for editing the manuscript.

\section{Authors' contribution}

Conceptualization, P.M, Z.H and S.H.N; Formal analysis, Z.H, F.H.E and S.H.N; Methodology, P.M and F.H.E; Supervision, F.A; Writing the original draft, P.M and Z.H; Writing review \& editing, Z.H and F.H.E. 


\section{Funding}

This study was supported by the Research Institute for Endocrine Sciences, Shahid Beheshti University of Medical Sciences, Tehran, Iran.

\section{Availability of data and materials}

The datasets used and/or analyzed during the current study are available from the corresponding author on reasonable request.

\section{Ethics approval and consent to participate}

The study was conducted based on the Declaration of Helsinki and the study protocol was accepted by the ethics committee of the Research Institute for Endocrine Sciences, Shahid Beheshti University of Medical Sciences, Tehran, Iran. All methods were performed in line with their relevant guidelines and regulations.

\section{Consent for publication}

Not applicable.

\section{Competing interests}

The authors have no conflicts of interest to declare.

\section{References}

1. World Health Organization Global Health Estimates 2016. [ Jul 2019]. Available from: http://www.who.int/healthinfo/global_burden_disease/estimates/en/index1.html.

2. Ceconi C, Boraso A, Cargnoni A, Ferrari R. Oxidative stress in cardiovascular disease: myth or fact? Archives of biochemistry and biophysics. 2003;420(2):217-21.

3. Mangge $H$, Becker K, Fuchs D, Gostner JM. Antioxidants, inflammation and cardiovascular disease. World journal of cardiology. 2014;6(6):462-77.

4. Jain AK, Mehra NK, Swarnakar NK. Role of Antioxidants for the Treatment of Cardiovascular Diseases: Challenges and Opportunities. Curr Pharm Des. 2015;21(30):4441-55.

5. Griffiths K, Aggarwal BB, Singh RB, Buttar HS, Wilson D, De Meester F. Food Antioxidants and Their AntiInflammatory Properties: A Potential Role in Cardiovascular Diseases and Cancer Prevention. Diseases (Basel, Switzerland). 2016;4(3).

6. Jayedi A, Rashidy-Pour A, Parohan M, Zargar MS, Shab-Bidar S. Dietary and circulating vitamin C, vitamin $\mathrm{E}$, beta-carotene and risk of total cardiovascular mortality: a systematic review and dose-response metaanalysis of prospective observational studies. Public health nutrition. 2019;22(10):1872-87.

7. Pfister R, Sharp SJ, Luben R, Wareham NJ, Khaw KT. Plasma vitamin C predicts incident heart failure in men and women in European Prospective Investigation into Cancer and Nutrition-Norfolk prospective study. American heart journal. 2011;162(2):246-53. 
8. Buijsse B, Jacobs DR, Jr., Steffen LM, Kromhout D, Gross MD. Plasma Ascorbic Acid, A Priori Diet Quality Score, and Incident Hypertension: A Prospective Cohort Study. PloS one. 2015;10(12):e0144920.

9. Monaghan BR, Schmitt FO. The effects of carotene and of vitamin A on the oxidation of linoleic acid. Journal of Biological Chemistry. 1932;96:387-95.

10. Olsen T, Vinknes KJ, Svingen GFT, Pedersen ER, Tell GS, Blomhoff R, et al. Cardiovascular disease risk associated with serum apolipoprotein B is modified by serum vitamin A. Atherosclerosis. 2017;265:32530.

11. Lourenco BH, Vieira LP, Macedo A, Nakasato M, Marucci Mde F, Bocchi EA. Nutritional status and adequacy of energy and nutrient intakes among heart failure patients. Arquivos brasileiros de cardiologia. 2009;93(5):541-8.

12. Tan $\mathrm{C}$, Chen $\mathrm{H}, \mathrm{Xia} \mathrm{C}$. The prediction of cardiovascular disease based on trace element contents in hair and a classifier of boosting decision stumps. Biological trace element research. 2009;129(1-3):9-19.

13. de Oliveira Otto MC, Alonso A, Lee DH, Delclos GL, Bertoni AG, Jiang R, et al. Dietary intakes of zinc and heme iron from red meat, but not from other sources, are associated with greater risk of metabolic syndrome and cardiovascular disease. The Journal of nutrition. 2012;142(3):526-33.

14. Kubota Y, Iso H, Date C, Kikuchi S, Watanabe Y, Wada Y, et al. Dietary intakes of antioxidant vitamins and mortality from cardiovascular disease: the Japan Collaborative Cohort Study (JACC) study. Stroke. 2011;42(6):1665-72.

15. Sun Q, Spiegelman D, van Dam RM, Holmes MD, Malik VS, Willett WC, et al. White rice, brown rice, and risk of type 2 diabetes in US men and women. Archives of internal medicine. 2010;170(11):961-9.

16. Mirmiran P, Esfahani FH, Mehrabi Y, Hedayati M, Azizi F. Reliability and relative validity of an FFQ for nutrients in the Tehran lipid and glucose study. Public health nutrition. 2010;13(5):654-62.

17. Momenan AA, Delshad M, Sarbazi N, REZAEI GN, Ghanbarian A, AZIZI F. Reliability and validity of the Modifiable Activity Questionnaire (MAQ) in an Iranian urban adult population. 2012.

18. Ainsworth BE, Haskell WL, Whitt MC, Irwin ML, Swartz AM, Strath SJ, et al. Compendium of physical activities: an update of activity codes and MET intensities. Medicine and science in sports and exercise. 2000;32(9 Suppl):S498-504.

19. Klein S, Allison DB, Heymsfield SB, Kelley DE, Leibel RL, Nonas C, et al. Waist circumference and cardiometabolic risk: a consensus statement from Shaping America's Health: Association for Weight Management and Obesity Prevention; NAASO, The Obesity Society; the American Society for Nutrition; and the American Diabetes Association. The American journal of clinical nutrition. 2007;85(5):1197-202.

20. Friedewald WT, Levy RI, Fredrickson DS. Estimation of the concentration of low-density lipoprotein cholesterol in plasma, without use of the preparative ultracentrifuge. Clinical chemistry. 1972;18(6):499502 .

21. Hadaegh F, Harati H, Ghanbarian A, Azizi F. Association of total cholesterol versus other serum lipid parameters with the short-term prediction of cardiovascular outcomes: Tehran Lipid and Glucose Study. European journal of cardiovascular prevention and rehabilitation : official journal of the European Society of Cardiology, Working Groups on Epidemiology \& Prevention and Cardiac Rehabilitation and Exercise Physiology. 2006;13(4):571-7. 
22. D'Agostino RB, Sr., Vasan RS, Pencina MJ, Wolf PA, Cobain M, Massaro JM, et al. General cardiovascular risk profile for use in primary care: the Framingham Heart Study. Circulation. 2008;117(6):743-53.

23. Williams B, Mancia G, Spiering W, Rosei EA, Azizi M, Burnier M, et al. [2018 ESC/ESH Guidelines for the management of arterial hypertension. The Task Force for the management of arterial hypertension of the European Society of Cardiology (ESC) and the European Society of Hypertension (ESH)]. Giornale italiano di cardiologia (2006). 2018;19(11):3-73.

24. Lee PH, Burstyn I. Identification of confounder in epidemiologic data contaminated by measurement error in covariates. BMC medical research methodology. 2016;16:54.

25. Rimm EB, Stampfer MJ, Ascherio A, Giovannucci E, Colditz GA, Willett WC. Vitamin E consumption and the risk of coronary heart disease in men. The New England journal of medicine. 1993;328(20):1450-6.

26. Stampfer MJ, Hennekens CH, Manson JE, Colditz GA, Rosner B, Willett WC. Vitamin E consumption and the risk of coronary disease in women. The New England journal of medicine. 1993;328(20):1444-9.

27. Buring JE, Hennekens CH. Antioxidant vitamins and cardiovascular disease. Nutrition reviews. 1997;55(1 Pt 2):S53-8; discussion S8-60.

28. Mathur P, Ding Z, Saldeen T, Mehta JL. Tocopherols in the Prevention and Treatment of Atherosclerosis and Related Cardiovascular Disease. Clinical cardiology. 2015;38(9):570-6.

29. Vardi M, Levy NS, Levy AP. Vitamin E in the prevention of cardiovascular disease: the importance of proper patient selection. Journal of lipid research. 2013;54(9):2307-14.

30. Sozen E, Demirel T, Ozer NK. Vitamin E: Regulatory role in the cardiovascular system. 2019;71(4):507-15.

31. American heart association. Vitamin Supplements: Hype or Help for Healthy Eating. 2019.

32. Martin-Calvo N, Martinez-Gonzalez MA. Vitamin C Intake is Inversely Associated with Cardiovascular Mortality in a Cohort of Spanish Graduates: the SUN Project. Nutrients. 2017;9(9).

33. d'Uscio LV, Milstien S, Richardson D, Smith L, Katusic ZS. Long-term vitamin C treatment increases vascular tetrahydrobiopterin levels and nitric oxide synthase activity. Circulation research. 2003;92(1):8895.

34. Salvayre R, Negre-Salvayre A, Camare C. Oxidative theory of atherosclerosis and antioxidants. Biochimie. 2016;125:281-96.

35. Siow RC, Richards JP, Pedley KC, Leake DS, Mann GE. Vitamin C protects human vascular smooth muscle cells against apoptosis induced by moderately oxidized LDL containing high levels of lipid hydroperoxides. Arteriosclerosis, thrombosis, and vascular biology. 1999;19(10):2387-94.

36. Myung SK, Ju W, Cho B, Oh SW, Park SM, Koo BK, et al. Efficacy of vitamin and antioxidant supplements in prevention of cardiovascular disease: systematic review and meta-analysis of randomised controlled trials. BMJ (Clinical research ed). 2013;346:f10.

37. Olson JA. Serum levels of vitamin A and carotenoids as reflectors of nutritional status. Journal of the National Cancer Institute. 1984;73(6):1439-44.

38. Chu A, Foster M, Samman S. Zinc Status and Risk of Cardiovascular Diseases and Type 2 Diabetes Mellitus-A Systematic Review of Prospective Cohort Studies. Nutrients. 2016;8(11).

39. Milton AH, Vashum KP, McEvoy M, Hussain S, McElduff P, Byles J. Prospective Study of Dietary Zinc Intake and Risk of Cardiovascular Disease in Women. 2018;10(1). 


\section{Tables}

Table 1. Baseline characteristics of adult participants of the Tehran Lipid and Glucose Study

\begin{tabular}{lllll}
\hline \multicolumn{1}{c}{ Total sample } & & Men & Women & $\mathrm{P}$ \\
& $\mathrm{N}=5102$ & $\mathrm{~N}=2253$ & $\mathrm{~N}=2849$ & \\
\hline Baseline age (years) & $46.2 \pm 11.1^{*}$ & $47.0 \pm 11.6$ & $45.6 \pm 10.5$ & $<0.001$ \\
Current smokers (\%) & 19.1 & 34.8 & 6.7 & $<0.001$ \\
Physical activity (MET/min/week) & $524 \pm 793$ & $588 \pm 844$ & $473 \pm 687$ & $<0.001$ \\
BMI (Kg/m2) & $28.1 \pm 4.5$ & $27.1 \pm 4.0$ & $28.8 \pm 4.8$ & $<0.001$ \\
Waist circumference (cm) & $93.8 \pm 11.0$ & $96.5 \pm 10.3$ & $91.8 \pm 11.4$ & $<0.001$ \\
SBP (mmHg) & $114 \pm 16.7$ & $118 \pm 15.9$ & $112 \pm 17.1$ & $<0.001$ \\
DBP (mmHg) & $76.3 \pm 10.2$ & $78.7 \pm 10.3$ & $74.4 \pm 10.2$ & $<0.001$ \\
Total cholesterol (mg/dl) & $219 \pm 121$ & $234 \pm 138$ & $207 \pm 117$ & $<0.001$ \\
LDL (mg/dl) & $118 \pm 31$ & $118 \pm 31$ & $118 \pm 32$ & 0.51 \\
TG/HDL-ratio & $3.8 \pm 3.0$ & $4.6 \pm 3.7$ & $3.1 \pm 2.4$ & $<0.001$ \\
FPG (mg/dl) & $98.1 \pm 26.6$ & $99.3 \pm 26.2$ & $97.2 \pm 27.0$ & 0.007 \\
2h- plasma glucose (mg/dl) & $108 \pm 43.1$ & $106 \pm 44.5$ & $110 \pm 40.3$ & 0.004 \\
Energy intake (kcal/day) & $2314 \pm 714$ & $2416 \pm 728$ & $2230 \pm 695$ & $<0.001$ \\
Carbohydrate (\% of energy) & $58.5 \pm 8.3$ & $59.9 \pm 6.4$ & $57.5 \pm 9.5$ & $<0.001$ \\
Protein (\% of energy) & $14.6 \pm 5.2$ & $14.4 \pm 2.7$ & $14.7 \pm 8.1$ & 0.19 \\
Total fat (\% of energy) & $30.4 \pm 10.3$ & $28.7 \pm 6.0$ & $31.8 \pm 17.6$ & $<0.001$ \\
SFA (\% of energy) & $10.1 \pm 11.1$ & $9.5 \pm 2.7$ & $10.6 \pm 16.9$ & 0.003 \\
MUFA (\% of energy) & $10.4 \pm 11.3$ & $9.7 \pm 2.6$ & $10.9 \pm 16.9$ & $<0.001$ \\
PUFA (\% of energy) & $6.3 \pm 12.1$ & $5.8 \pm 1.9$ & $6.7 \pm 16.9$ & $<0.001$ \\
Fiber (g/1000 kcal) & $9.7 \pm 3.3$ & $9.0 \pm 2.8$ & $10.2 \pm 3.8$ & $<0.001$ \\
Vitamin E (mg/day) & $12.1 \pm 5.3$ & $11.2 \pm 5.0$ & $12.8 \pm 44.5$ & 0.08 \\
Vitamin C (mg/day) & $170 \pm 123$ & $160 \pm 108$ & $178 \pm 130$ & $<0.001$ \\
Vitamin A (pg/day) & $600 \pm 371$ & $565 \pm 350$ & $629 \pm 399$ & $<0.001$ \\
Zinc (mg/day) & $13.7 \pm 26.3$ & $14.0 \pm 12.4$ & $13.4 \pm 45.1$ & 0.58 \\
\hline
\end{tabular}

MET: Metabolic Equivalent, BMI: body mass index, SBP: systolic blood pressure, DBP: diastolic blood pressure, MUFA: Mono-unsaturated fatty acids. PUFA: Poly-unsaturated fatty acids; SFA, Saturated fat

*values are mean \pm SD unless otherwise listed

P-values derived through Student's t-test and chi-square test for quantitative and qualitative variables, respectively

Table 2. Antioxidants intakes with respect to quartiles of food groups' consumption 


\begin{tabular}{|c|c|c|c|c|}
\hline Food consumption & $\begin{array}{c}\text { Vitamin }_{(\mathrm{mg})} \mathrm{E} \\
\text {. }\end{array}$ & $\begin{array}{c}\operatorname{Vitamin}_{(\mathrm{mg})} \mathrm{C} \\
\end{array}$ & $\begin{array}{c}\text { Vitamin A } \\
(\mu \mathrm{g})\end{array}$ & $\begin{array}{l}\text { Zinc } \\
\text { (mg) }\end{array}$ \\
\hline \multicolumn{5}{|l|}{ Fruits } \\
\hline$Q 1: 0.70 \pm 0.27^{b}$ & $9.7 \pm 4.7$ & $79.7 \pm 44.1$ & $421 \pm 256$ & $11.3 \pm 8.1$ \\
\hline Q2: $1.60 \pm 0.27$ & $10.8 \pm 10.8$ & $123 \pm 36.4$ & $534 \pm 320$ & $12.9 \pm 10.3$ \\
\hline Q3: 2.69 & $14.0 \pm 66.2$ & $178 \pm 89.0$ & $625 \pm 351$ & $15.3 \pm 66.5$ \\
\hline Q4: $5.59 \pm 2.55$ & $14.1 \pm 5.0$ & $300 \pm 137$ & $822 \pm 446$ & $15.1 \pm 14.3$ \\
\hline$P$ for trend ${ }^{c}$ & $<0.001$ & $<0.001$ & $<0.001$ & 0.01 \\
\hline \multicolumn{5}{|l|}{ Vegetables } \\
\hline 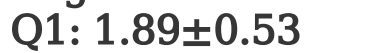 & $9.1 \pm 4.5$ & $101 \pm 83.4$ & $357 \pm 212$ & $10.5 \pm 8.7$ \\
\hline Q2: 3.28 & $11.0 \pm 4.5$ & $145 \pm 94.4$ & $492 \pm 213$ & $12.2 \pm 7.9$ \\
\hline Q3: 4.57 & $12.3 \pm 5.3$ & $183 \pm 92.5$ & $632 \pm 278$ & $13.4 \pm 10.3$ \\
\hline Q4: 7.66士 & $16.1 \pm 66.2$ & $251 \pm 142$ & $920 \pm 484$ & $18.5 \pm 67.3$ \\
\hline$P$ for tren & $<0.001$ & $<0.001$ & $<0.001$ & $<0.001$ \\
\hline \multicolumn{5}{|l|}{ Legumes } \\
\hline Q1: $0.02 \pm$ & $11.6 \pm 5.3$ & $157 \pm 126$ & $514 \pm 341$ & $11.6 \pm 6.7$ \\
\hline Q2: 0.07士 & $11.8 \pm 5.6$ & $172 \pm 122$ & $598 \pm 389$ & $13.0 \pm 10.6$ \\
\hline Q3: 0.15 & $11.5 \pm 4.8$ & $167 \pm 107$ & $613 \pm 360$ & $13.7 \pm 12.6$ \\
\hline Q4: 0.35 & $13.6 \pm 8.2$ & $183 \pm 132$ & $677 \pm 407$ & $16.4 \pm 67.0$ \\
\hline$P$ for tr & $<0.001$ & & $<0.001$ & $<0.001$ \\
\hline \multicolumn{5}{|l|}{ Whole grains } \\
\hline$Q 1: 0.10 \pm 0.07$ & $11.2 \pm 5.2$ & $149 \pm 118$ & $511 \pm 344$ & $11.3 \pm 8.3$ \\
\hline Q2: 0.44士 & $11.6 \pm 5.5$ & $167 \pm 107$ & $599 \pm 386$ & $12.5 \pm 8.7$ \\
\hline Q3: $1.31=$ & $13.5 \pm 66.2$ & $175 \pm 131$ & $622 \pm 385$ & $14.1 \pm 13.7$ \\
\hline Q4: 5.01士 & $12.5 \pm 4.8$ & $189 \pm 123$ & $669 \pm 384$ & $16.7 \pm 66.9$ \\
\hline & & & -0001 & 0.002 \\
\hline \multicolumn{5}{|l|}{ Refined grains } \\
\hline & $10.6 \pm 5.7$ & $173 \pm 126$ & $614 \pm 432$ & $11.0 \pm 8.1$ \\
\hline Q2: $7.12 \pm 0.72$ & $11.3 \pm 5.1$ & $168 \pm 111$ & $591 \pm 347$ & $12.3 \pm 10.9$ \\
\hline Q3: $10.0 \pm$ & $12.0 \pm 5.0$ & $173 \pm 118$ & $607 \pm 378$ & $14.0 \pm 12.8$ \\
\hline Q4: $16.6 \pm$ & $14.6 \pm 66.2$ & $166 \pm 129$ & $590 \pm 356$ & $17.4 \pm 66.7$ \\
\hline & & 0007 & 0004 & $<0.001$ \\
\hline \multicolumn{5}{|l|}{ Dairy products } \\
\hline & $12.3 \pm 66.3$ & $137 \pm 119$ & $449 \pm 325$ & $12.5 \pm 67.4$ \\
\hline Q2: $1.69 \pm 0.18$ & $11.2 \pm 4.6$ & & $544 \pm 332$ & $12.2 \pm 7.8$ \\
\hline Q3: $2.35 \pm 0.22$ & $11.9 \pm 5.0$ & $181 \pm 125$ & $624 \pm 344$ & $13.6 \pm 9.4$ \\
\hline Q4: $3.71 \pm 1.07$ & $13.1 \pm 5.5$ & $201 \pm 123$ & $785 \pm 326$ & $16.2 \pm 11.0$ \\
\hline$P$ for trend & 0.19 & $<0.001$ & $<0.001$ & $<0.001$ \\
\hline
\end{tabular}




\begin{tabular}{|c|c|c|c|c|}
\hline Food consumption & $\begin{array}{c}\text { Vitamin }_{(\mathrm{mg})} \\
\mathrm{V}\end{array}$ & $\underset{(\mathrm{mg})}{\text { Vitamin C }^{\text {intan }}}$ & $\begin{array}{c}{ }_{(\mu g)} \\
\text { Vitamin A }\end{array}$ & $\begin{array}{l}\text { Zinc } \\
\text { (mg) }\end{array}$ \\
\hline $\begin{array}{l}\text { Red meat } \\
\text { Q1: } 0.07 \pm 0.03 \\
\text { Q2: } 0.16 \pm 0.02 \\
\text { Q3: } 0.27 \pm 0.04 \\
\text { Q4: } 0.60 \pm 0.32 \\
\text { P for trend }\end{array}$ & $\begin{array}{c}12.0 \pm 5.4 \\
11.7 \pm 4.9 \\
13.4 \pm 66.2 \\
11.5 \pm 5.6 \\
0.70\end{array}$ & $\begin{array}{c}181 \pm 140 \\
172 \pm 120 \\
168 \pm 122 \\
159 \pm 98.6 \\
<0.001\end{array}$ & $\begin{array}{c}612 \pm 404 \\
602 \pm 349 \\
595 \pm 344 \\
593 \pm 416 \\
0.12\end{array}$ & $\begin{array}{c}13.3 \pm 9.2 \\
13.4 \pm 12.0 \\
14.3 \pm 66.3 \\
13.8 \pm 13.8 \\
0.32\end{array}$ \\
\hline $\begin{array}{l}\text { Fish and poultry } \\
\text { Q1: } 0.41 \pm 0.15 \\
\text { Q2: } 0.84 \pm 0.13 \\
\text { Q3: } 1.32 \pm 0.16 \\
\text { Q4: } 2.92 \pm 1.87 \\
\text { P for trend }\end{array}$ & $\begin{array}{c}10.7 \pm 4.9 \\
11.2 \pm 4.9 \\
12.1 \pm 5.0 \\
14.6 \pm 66.3 \\
0.001\end{array}$ & $\begin{array}{c}148 \pm 116 \\
161 \pm 112 \\
174 \pm 112 \\
197 \pm 137 \\
<0.001\end{array}$ & $\begin{array}{c}499 \pm 346 \\
554 \pm 318 \\
627 \pm 386 \\
722 \pm 498 \\
<0.001\end{array}$ & $\begin{array}{c}11.3 \pm 8.3 \\
12.5 \pm 8.7 \\
14.1 \pm 13.7 \\
16.7 \pm 66.9 \\
<0.001\end{array}$ \\
\hline $\begin{array}{l}\text { Sugar-sweetened soft drinks }(\mathrm{ml} / \mathrm{d}) \\
\text { Q1: } 0.76 \pm 0.95 \\
\text { Q2: } 8.29 \pm 2.23 \\
\text { Q3: } 24.3 \pm 7.59 \\
\text { Q4: } 109 \pm 101 \\
\text { P for trend }\end{array}$ & $\begin{array}{c}13.1 \pm 66.1 \\
11.1 \pm 4.6 \\
11.7 \pm 4.8 \\
12.6 \pm 5.7 \\
0.55\end{array}$ & $\begin{array}{c}170 \pm 131 \\
159 \pm 117 \\
174 \pm 122 \\
176 \pm 113 \\
<0.001\end{array}$ & $\begin{array}{c}595 \pm 382 \\
560 \pm 376 \\
622 \pm 380 \\
626 \pm 377 \\
<0.001\end{array}$ & $\begin{array}{c}14.2 \pm 66.4 \\
12.7 \pm 11.2 \\
14.1 \pm 14.4 \\
13.8 \pm 7.6 \\
0.68\end{array}$ \\
\hline $\begin{array}{l}\text { Vegetable oils } \\
\text { Q1: } 2.13 \pm 1.25 \\
\text { Q2: } 6.27 \pm 0.73 \\
\text { Q3: } 9.26 \pm 1.31 \\
\text { Q4: } 18.62 \pm 8.98 \\
\text { P for trend }\end{array}$ & $\begin{array}{c}8.4 \pm 3.5 \\
10.6 \pm 3.5 \\
13.7 \pm 65.9 \\
16.1 \pm 6.4 \\
0.04\end{array}$ & $\begin{array}{c}138 \pm 98 \\
163 \pm 116 \\
184 \pm 141 \\
196 \pm 118 \\
0.07\end{array}$ & $\begin{array}{c}497 \pm 311 \\
563 \pm 341 \\
641 \pm 392 \\
703 \pm 434 \\
0.04\end{array}$ & $\begin{array}{c}11.7 \pm 9.8 \\
13.0 \pm 13.2 \\
15.5 \pm 66.5 \\
14.5 \pm 9.3 \\
0.30\end{array}$ \\
\hline $\begin{array}{l}\text { Nuts } \\
\text { Q1: } 0.02 \pm 0.01 \\
\text { Q2: } 0.08 \pm 0.01 \\
\text { Q3: } 0.17 \pm 0.03 \\
\text { Q4: } 0.55 \pm 0.42 \\
\text { P for trend }\end{array}$ & $\begin{array}{c}11.2 \pm 5.1 \\
13.6 \pm 66.2 \\
11.7 \pm 11.7 \\
12.0 \pm 5.6 \\
0.96\end{array}$ & $\begin{array}{c}145 \pm 109 \\
169 \pm 135 \\
177 \pm 113 \\
188 \pm 188 \\
<0.001\end{array}$ & $\begin{array}{c}537 \pm 379 \\
596 \pm 364 \\
621 \pm 373 \\
648 \pm 393 \\
<0.001\end{array}$ & $\begin{array}{c}12.5 \pm 108 \\
15.0 \pm 66.7 \\
13.4 \pm 11.7 \\
13.8 \pm 10.7 \\
0.33\end{array}$ \\
\hline $\begin{array}{l}\text { Tea and coffee }(\mathrm{ml} / \mathrm{d}) \\
\text { Q1: } 200 \pm 88.1 \\
\text { Q2: } 379 \pm 112 \\
\text { Q3: } 679 \pm 101 \\
\text { Q4: } 1342 \pm 741 \\
\text { P for trend }\end{array}$ & $\begin{array}{c}12.7 \pm 66.4 \\
11.5 \pm 4.8 \\
12.0 \pm 4.6 \\
12.5 \pm 6.3 \\
0.50\end{array}$ & $\begin{array}{c}156 \pm 123 \\
169 \pm 118 \\
175 \pm 121 \\
179 \pm 121 \\
<0.001\end{array}$ & $\begin{array}{c}549 \pm 359 \\
612 \pm 398 \\
613 \pm 342 \\
628 \pm 383 \\
0.001\end{array}$ & $\begin{array}{c}14.5 \pm 67.2 \\
13.1 \pm 11.2 \\
13.5 \pm 11.2 \\
13.6 \pm 7.8 \\
0.97\end{array}$ \\
\hline
\end{tabular}

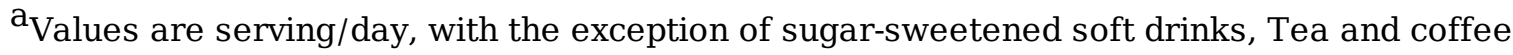

${ }^{\mathrm{b}}$ Data are mean \pm SD unless otherwise listed

${ }^{\mathrm{C}} \mathrm{P}$ for trend obtained through linear regression analysis via the mean of each quartile as a continuous variable for each food group
}

Table 3. Hazard Ratios (HR) and 95\% CIs for cardiovascular diseases according to quartile dietary antioxidants intake. 


\begin{tabular}{|c|c|c|c|c|c|}
\hline \multirow[b]{2}{*}{ Variables } & \multicolumn{5}{|c|}{ Quartiles of intake } \\
\hline & Q1 & Q2 & Q3 & Q4 & $\mathrm{P}_{\text {trend }^{a}}{ }^{a}$ \\
\hline Vitamin $\mathrm{E}$ intake & & & & & \\
\hline Median, mg/d & 6.8 & 9.5 & 12.4 & 16.8 & \\
\hline Cases, $\mathbf{n}$ & 61 & 56 & 51 & 38 & \\
\hline Person-years, n & 6711 & 6530 & 6593 & 6423 & \\
\hline Follow-up, y & 5.3 & 5.2 & 5.2 & 5.1 & \\
\hline Incidence, \% & 9.0 & 8.5 & 7.7 & 5.9 & \\
\hline Crude & 1.00 ref. & $0.94(0.65-1.35)$ & $0.85(0.58-1.23)$ & $0.65(0.43-0.98)$ & 0.03 \\
\hline Model adjusted ${ }^{\mathbf{b}}$ & 1.00 ref. & $0.91(0.62-1.64)$ & $0.77(0.51-1.18)$ & $0.57(0.34-0.97)$ & 0.03 \\
\hline \multicolumn{6}{|l|}{ Vitamin C intake } \\
\hline Median, mg/d & 64.7 & 114.2 & 173.3 & 291.6 & \\
\hline Cases, $\mathrm{n}$ & 55 & 52 & 63 & 46 & \\
\hline Person-years, n & 6844 & 6643 & 6515 & 6256 & \\
\hline Follow-up, y & 5.5 & 5.3 & 5.1 & 5.0 & \\
\hline Incidence, \% & 6.5 & 7.8 & 9.6 & 7.3 & \\
\hline Crude & 1.00 ref. & $1.19(0.80-1.77)$ & $1.47(1.00-2.16)$ & $1.13(0.74-1.70)$ & 0.58 \\
\hline Model adjusted $\mathrm{b}$ & 1.00 ref. & $1.04(0.68-1.59)$ & $1.19(0.76-1.85)$ & $0.84(0.48-1.48)$ & 0.44 \\
\hline \multicolumn{6}{|l|}{ Vitamin A intake } \\
\hline Median, $\mu \mathrm{g} / \mathrm{d}$ & 267 & 428 & 611 & 964 & \\
\hline Cases, $\mathbf{n}$ & 53 & 60 & 41 & 52 & \\
\hline Person-years, n & 7020 & 6020 & 6472 & 6145 & \\
\hline Follow-up, y & 5.7 & 5.3 & 5.1 & 5.0 & \\
\hline Incidence, \% & 7.5 & 9.0 & 6.3 & 8.4 & \\
\hline Crude & 1.00 ref. & $1.21(0.83-1.75)$ & $0.84(0.56-1.27)$ & $1.13(0.77-1.67)$ & 0.81 \\
\hline Model adjusted ${ }^{b}$ & 1.00 ref. & $1.16(0.78-1.70)$ & $0.82(0.52-1.28)$ & $1.00(0.61-1.63)$ & 0.74 \\
\hline \multicolumn{6}{|l|}{ Zinc } \\
\hline Median intake (mg/d) & 7.6 & 10.5 & 13.2 & 17.8 & \\
\hline Cases, $\mathbf{n}$ & 51 & 61 & 41 & 53 & \\
\hline Person-years, n & 6959 & 6728 & 6409 & 6161 & \\
\hline Follow-up, y & 5.6 & 5.3 & 5.1 & 5.0 & \\
\hline Incidence, \% & 7.3 & 9.0 & 6.3 & 8.6 & \\
\hline Crude & 1.00 ref. & $1.24(0.85-1.80)$ & $0.88(0.58-1.33)$ & $1.16(0.81-1.76)$ & 0.62 \\
\hline Model adjusted b & 1.00 ref. & $1.20(0.80-1.81)$ & $0.81(0.49-1.35)$ & $1.09(0.58-2.05)$ & 0.97 \\
\hline
\end{tabular}

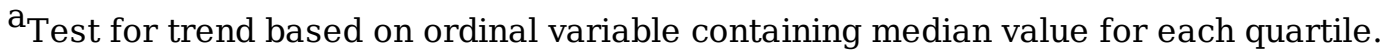

${ }^{\mathrm{b}}$ Adjusted for age, sex, CVD risk score, family history of CVD, physical activity, total energy intake, fiber and tota intakes
}

Figures 


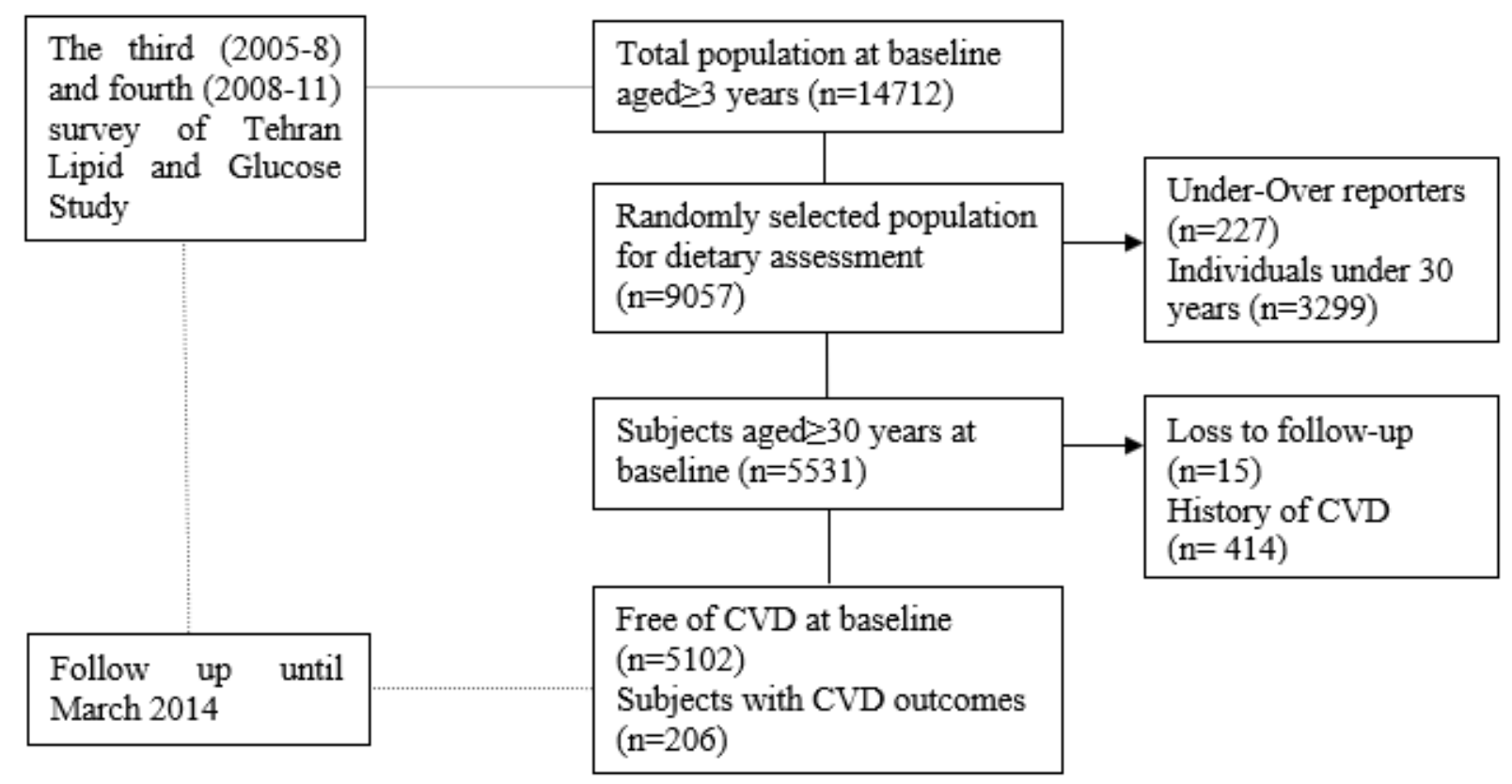

Figure 1

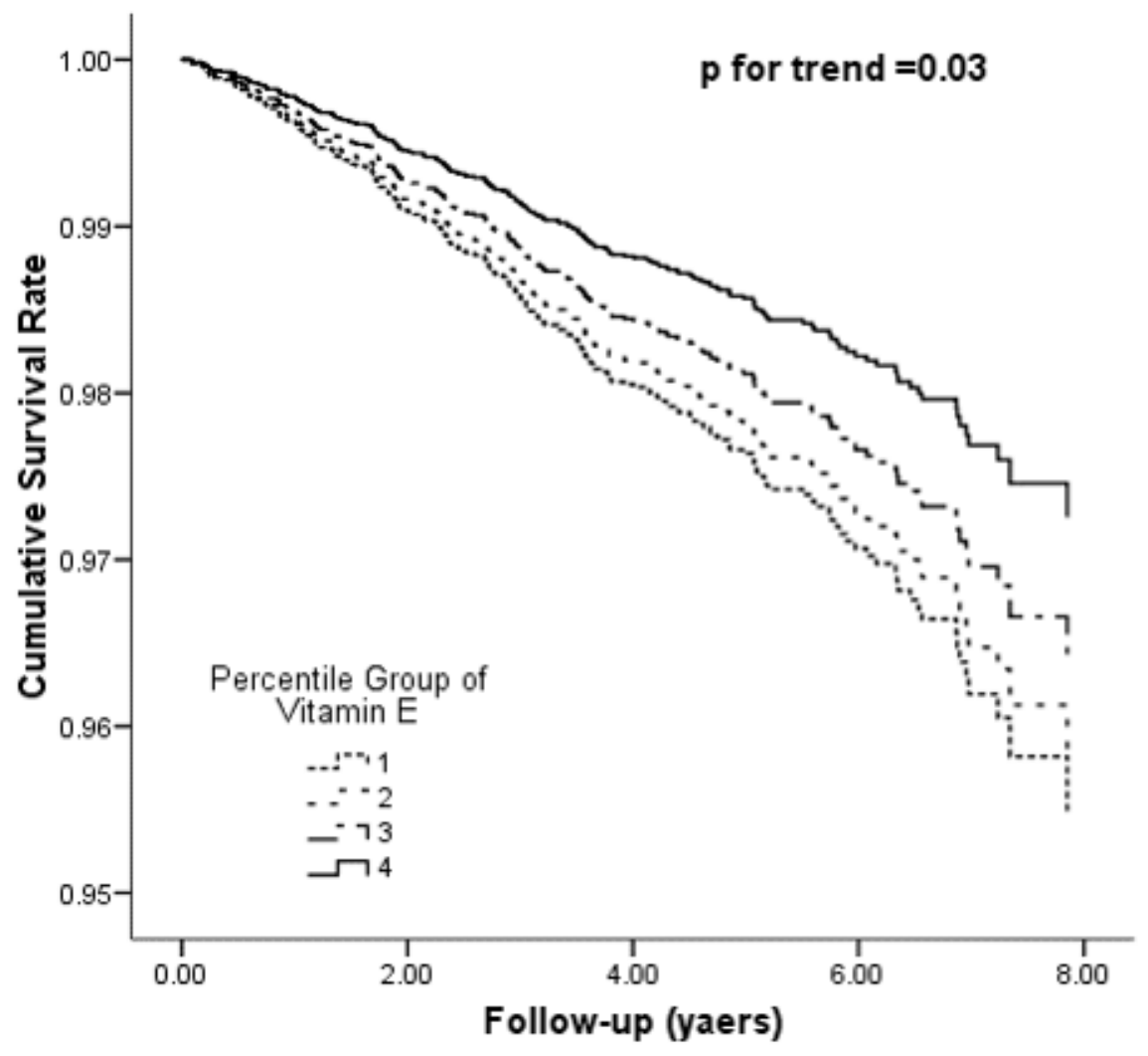

Figure 2 
Multivariable-adjusted cumulative survival curves for the incidence of CVD according to vitamin E categories. The multivariable-adjusted model included age, sex, CVD risk score, family history of CVD, physical activity, total energy intake, fiber and total fat intakes. The associations between the risk of CVD and quartiles of vitamin $\mathrm{E}$ intake were significant.

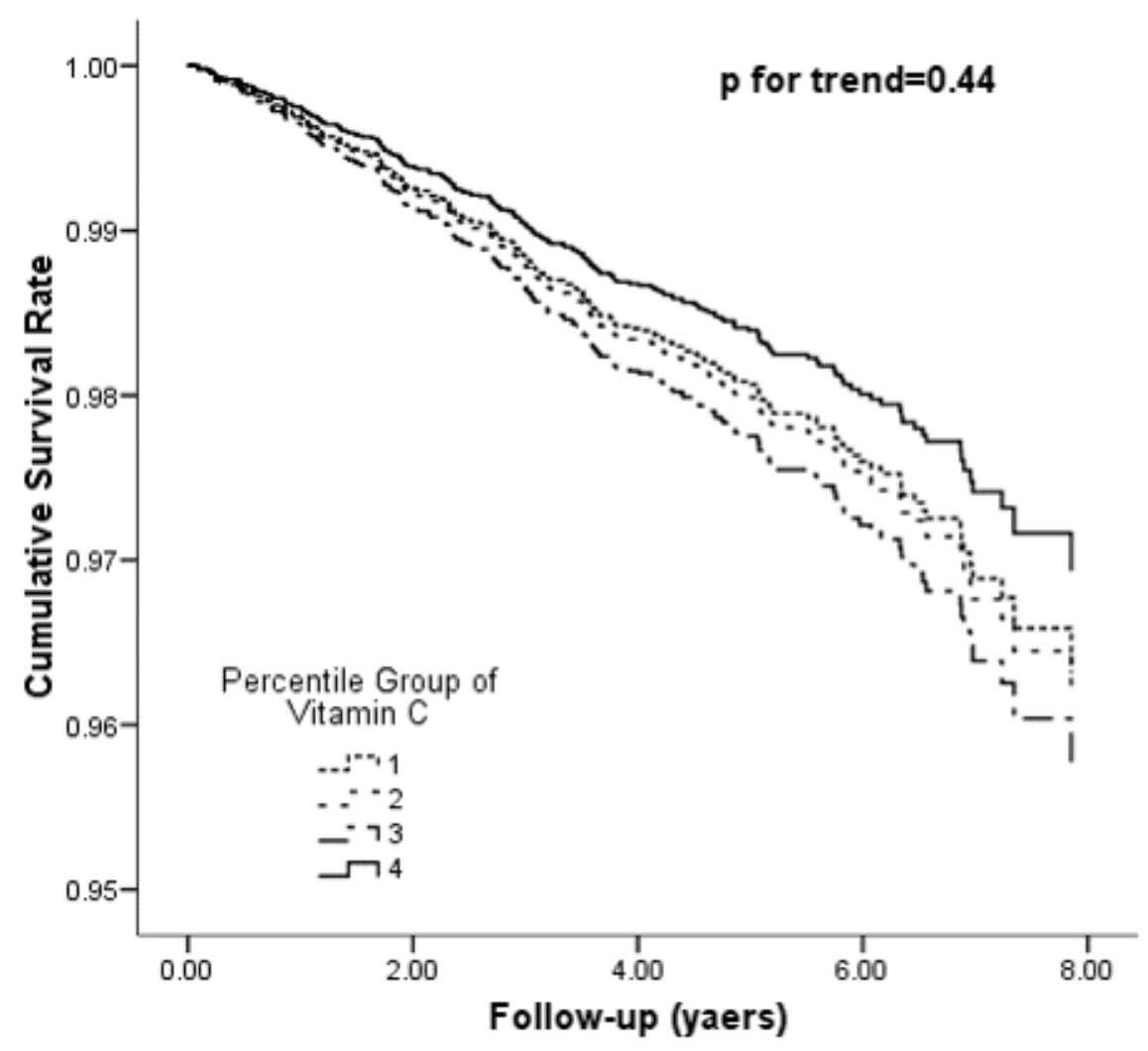

\section{Figure 3}

Multivariable-adjusted cumulative survival curves for the incidence of CVD according to vitamin C categories. The multivariable-adjusted model included age, sex, CVD risk score, family history of CVD, physical activity, total energy intake, fiber and total fat intakes. The associations between the risk of CVD and quartiles of vitamin $\mathrm{C}$ intake were not significant. 


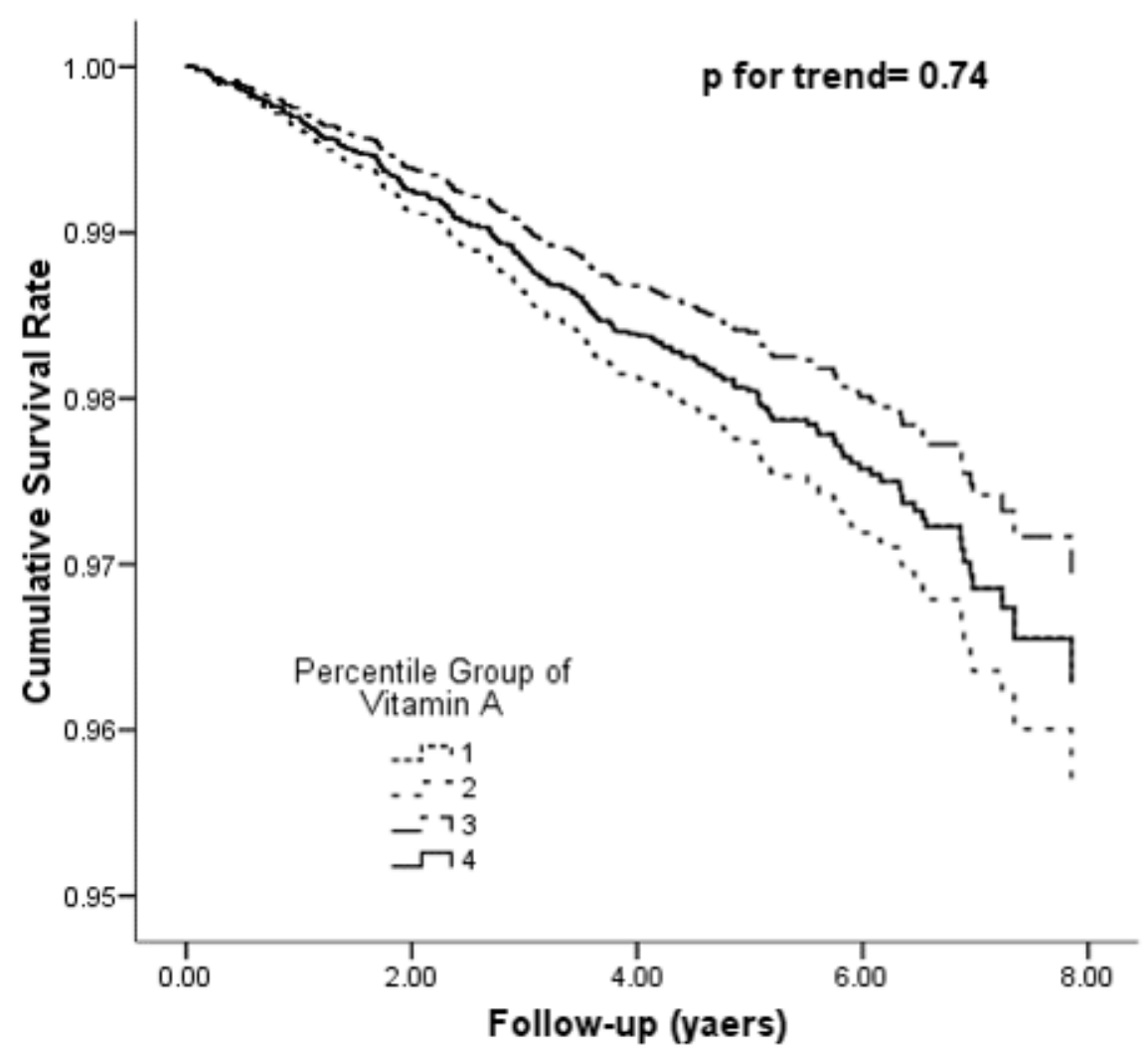

Figure 4

Multivariable-adjusted cumulative survival curves for CVD of diabetes according to vitamin A categories. The multivariable-adjusted model included age, sex, CVD risk score, family history of CVD, physical activity, total energy intake, fiber and total fat intakes. Associations between the risk of CVD and quartiles of vitamin A intake were not significant. 


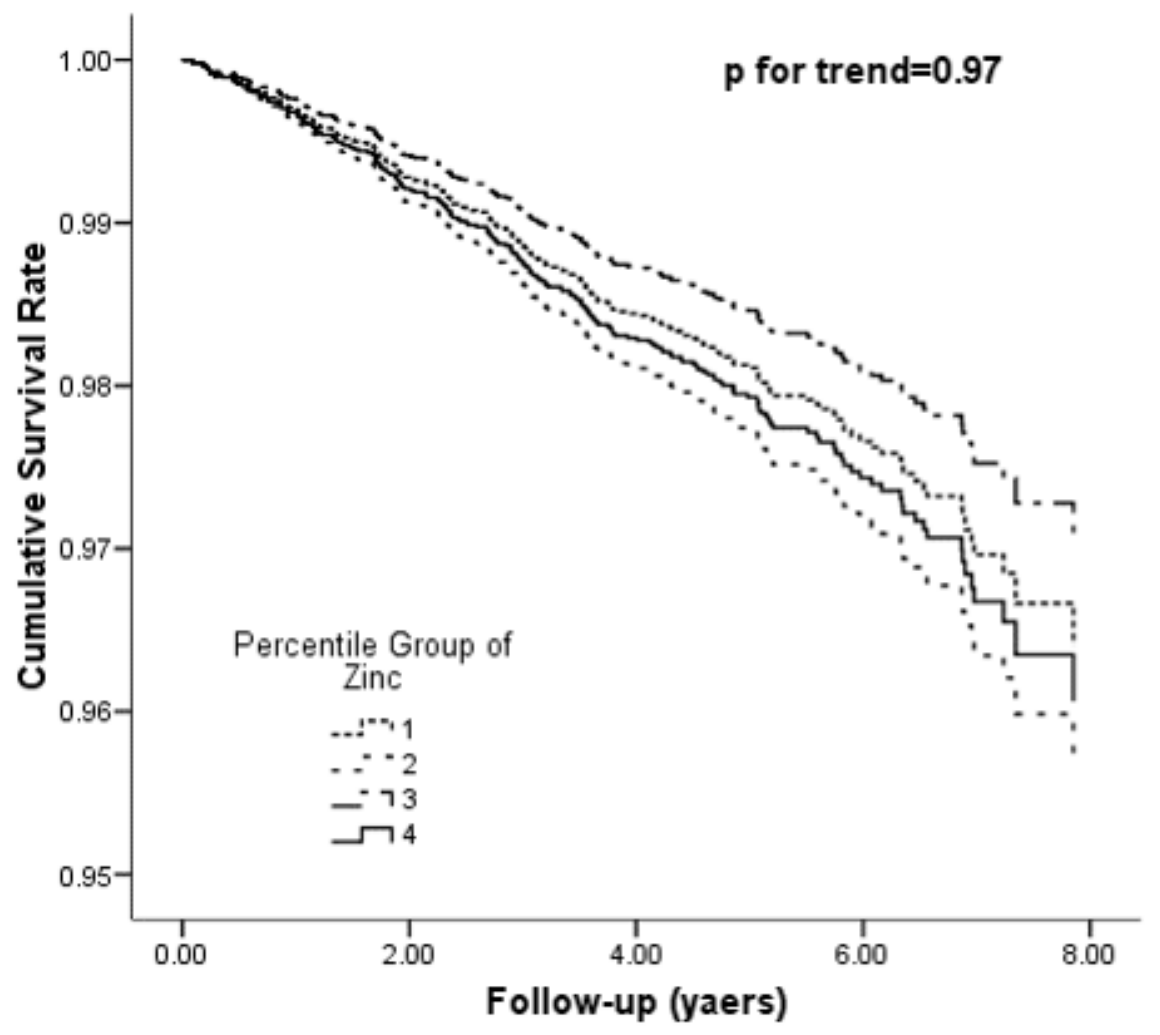

\section{Figure 5}

Multivariable-adjusted cumulative survival curves for CVD of diabetes according to zinc categories. The multivariable-adjusted model included age, sex, CVD risk score, family history of CVD, physical activity, total energy intake, fiber and total fat intakes. The associations between the risk of CVD and quartiles of zinc intake were not significant. 